\title{
Robust Direction of Arrival Estimation Using Uniform Circular Antenna Array based on Total Forward - Backward Matrix Pencil Method
}

\author{
Han Trong Thanh ${ }^{1}$, Do Trong Tuan ${ }^{1}$, Nguyen Trong Duc ${ }^{2}$ and Vu Van Yem ${ }^{1}$ \\ 1 'School of Electronics and Telecommunications, Hanoi University of Science and Technology, \\ No.1, Dai Co Viet Road, Hai Ba Trung District, Hanoi, Vietnam.
}

Abstract-In this paper, we propose an approach to estimate the Direction of Arrival (DOA) of Radio coherent incoming signals using the Total Forward Backward Matrix Pencil algorithm (TFBMP). This algorithm works directly on samples of signals impinging on an $\mathbf{M}$ - element Uniform Circular Antenna (UCA) array, which has a smaller size as well as larger observation angle in comparison with the Uniform Linear Antenna (ULA) array. Therefore, the correlation between the received signals does not significantly impact on its performance and efficiency. Furthermore, this algorithm can also extract the DOA information with only one snapshot of signal. Simulation results for DOA estimation using the proposed approach for different situations of incoming signals as well as the number of snapshots in the presence of noise will be assessed to verify its performance.

Keywords - Direction of Arrival (DOA), Total Forward Backward Matrix Pencil (TFBMP), Uniform Circular Antenna (UCA) Array.

\section{INTRODUCTION}

Radio Direction Finding (DF) is a technique that identifies the bearing angle or the coordinates of incoming radio signals. The most important information estimated by a DF system is the Direction of Arrival (DOA). DF systems have many applications in Radio Navigation, Emergency Aid and intelligent operations, etc. Several techniques to calculate the DOA information have been investigated. They can be classified into two main categories: amplitude comparison based [1] and phase comparison based [2], [3]. Some other algorithms can be the hybrid of the two such as the Correlative vector (CV) method [4].
In [5 - 8], the Matrix Pencil algorithm was proposed as a high - resolution technique for DOA estimation. Matrix Pencil has some advantages in comparison with the other super - resolution methods for DOA estimation such as MUSIC [9], which generally has to calculate the signal covariance matrix. Unlike other algorithms, Matrix Pencil works directly on signal samples and does not require independent data samples. Furthermore, the Matrix Pencil algorithm offers some benefits such as less processing power and faster executing than some other super - resolution methods [7]. One of the most remarkable advantages of this technique is that it can extract the DOA information with one snapshot.

Total Forward - Backward Matrix Pencil (TFBMP) is an extension of the Matrix Pencil Method. Total Forward - Backward is the preprocessing technique to break the correlative property of received signals. This fact helps the Matrix Pencil method to estimate the DOA information of coherent incoming signals. Although TFBMP deals with a larger database, however it is more efficient than the original method, especially for a multipath environment. TFBMP was utilized for the high resolution frequency estimator. In some scenarios, it provides better estimation results than the other methods such as Fourier technique [10].

In Conventional DF systems, there are two common types of the antenna array: Uniform Circular Antenna (UCA) and Uniform Linear Antenna (ULA). In general, with the same number of elements and the same spacing between adjacent radiators, UCA has a smaller size than ULA. Moreover, by using the UCA 


\section{Research, Development on Information and Communications Technology}

array, the angle of arrival of incoming signals can be determined from all directions in the azimuth plane. Meanwhile, when using the ULA array, the DOA information is only estimated from $-90^{\circ}$ to $90^{\circ}$. That is why the UCA is of very practical interest and is often adopted in radar and sonar systems as well as in communication system for 3D channel modeling purpose.

In this paper, we develop an approach based on the TFBMP technique to solve the DOA estimation problem using an $\mathrm{M}$ - element Uniform Circular Antenna (UCA) array. The performance of this method will be assessed in many cases that depend on the characteristics of incoming signals as well as number of snapshots of data.

The paper is organized as follows. Section II describes the model of the incoming signals based on phase mode excitation beam - forming. In Section III, we present in detail the TFBMP technique for DOA estimation. Simulation results are shown in Section IV. Conclusion is given in Section V.

\section{SIGNAL MODEL BASED ON PHASE MODE EXCITATION BEAMFORMING}

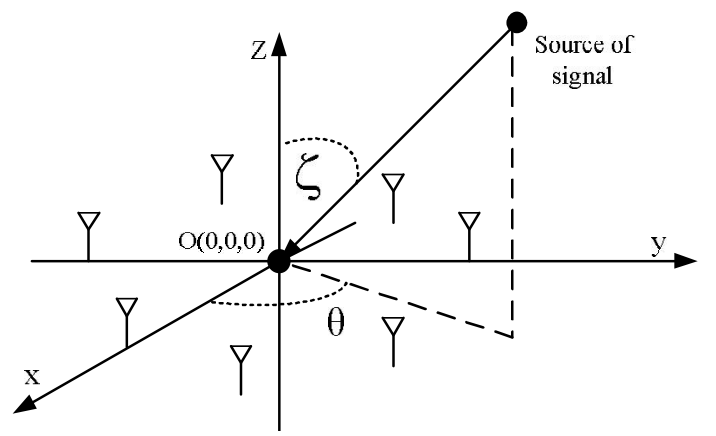

Fig. 1. Antenna array in the coordinate system

In our research, we utilize the Uniform Circular Antenna Array (UCA) model, which includes a set of $M$ isotropic antenna elements spaced around a circle with fixed radius $\mathrm{R}$. We have defined the reference point as the origin of the three - Dimensional Cartesian - coordinate system shown in Fig.1.

Assuming all signals impinging on the array are narrowband signals. For an $\mathrm{M}$ - element array depicted in Fig. 1, let $\theta$ be the DOA in azimuth, $\zeta$ be the DOA in elevation. The phase difference between any antenna and the reference point is given by

$\Delta \Psi_{m}=\beta\left(x_{m} \cos \theta \sin \zeta+y_{m} \sin \theta \sin \zeta+z_{m} \cos \zeta\right),(1)$

where $x_{m}, y_{m}, z_{m}$ are the three - dimensional coordinate of each antenna element and $\beta=\frac{2 \pi}{\lambda}$ is the propagation factor. Defining the array - manifold vector for an $\mathrm{M}$ - element array as

$$
a(\theta, \zeta)=\left[a_{0}(\theta, \zeta), a_{1}(\theta, \zeta) \ldots a_{M-1}(\theta, \zeta)\right]^{T},
$$

where $a_{m}$ is the phase response at antenna $m^{\text {th }}$ relative to the reference point:

$$
a_{m}=g_{m} e^{-j \Delta \Psi \mathrm{m}} .
$$

The single signal received by the array is $y(t)=$ $s(t) a(\theta, \zeta)$, where $s(t)$ is the complex baseband envelope of an incoming signal and $g_{m}$ is the gain of antenna. Adding receiver noise and allowing $K$ sources, the signal model for the data snapshots at time index is

$$
\boldsymbol{Y}=\boldsymbol{\Lambda} \boldsymbol{S}+\mathcal{\varepsilon},
$$

where

$$
\begin{gathered}
\boldsymbol{\Lambda}=\left[a\left(\theta_{1}, \zeta_{1}\right), a\left(\theta_{2}, \zeta_{2}\right), \ldots a\left(\theta_{K}, \zeta_{K}\right)\right], \\
\boldsymbol{S}=\left[s_{1}(t), s_{2}(t), s_{3}(t) \ldots s_{K}(t)\right]^{T},
\end{gathered}
$$

$\mathcal{E}$ is a noise vector representing AWGN in the received signal path and is assumed to be independent for each antenna and receiver path. In this paper, we only deal with the signal coming on the same plane of the array. Therefore, $\zeta=90^{\circ}$ and $z=0$. If it is assumed that spatial coordinates of a Uniform Circular Array with M elements are

$$
\begin{aligned}
& x_{m}=R \cos \left(\frac{2 \pi m}{M}\right), \\
& y_{m}=R \sin \left(\frac{2 \pi m}{M}\right),
\end{aligned}
$$

and according to Eq.1, Eq.3, Eq.7 and Eq.8, the single signal collected by $m^{\text {th }}$ antenna element is given by 


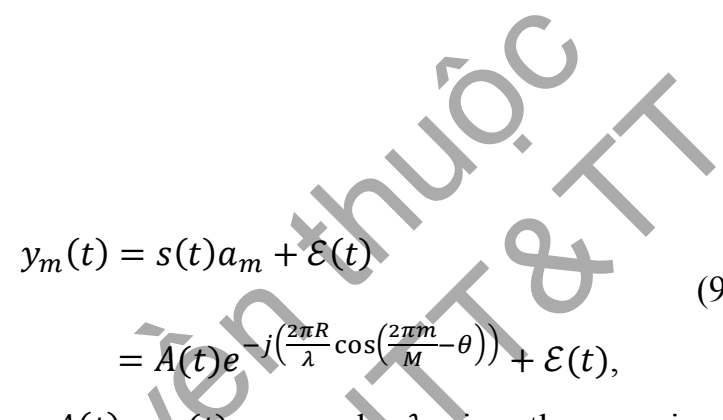

where $A(t)=s(t) g_{m}$ and $\lambda$ is the carrier wavelength.

In practice, there are several radio signals crossing the antenna array simultaneously. The received signal at each antenna element will be the sum of all arriving radio signals. In case of $K$ signals approaching the array from some azimuth directions $\theta_{1}, \theta_{2} \ldots \theta_{k}$, the received signal at the $m^{\text {th }}$ antenna is

$y_{m}(t)=\sum_{k=1}^{K} s_{k}(t) g_{m} e^{-j \frac{2 \pi R}{\lambda} \cos \left(\theta_{k}-\frac{2 \pi m}{M}\right)}+\mathcal{E}(t)$

$$
=\sum_{k=1}^{K} A_{k}(t) e^{-j \frac{2 \pi R}{\lambda} \cos \left(\theta_{k}-\frac{2 \pi m}{M}\right)}+\mathcal{E}(t),
$$

where $s_{k}(t)$ is the complex baseband envelope of an incoming signal at direction $\theta_{k}$.

This signal can then be sampled with period $T_{s}$ at discrete time instants $n T_{s}$. The discrete sampled form of the output signal at each antenna element is given as

$$
y_{m}(n)=\sum_{k=1}^{K} A_{k}(n) e^{-j \frac{2 \pi R}{\lambda} \cos \left(\theta_{k}-\frac{2 \pi m}{M}\right)}+\mathcal{E}(n) .
$$

Each sample of the output signal is defined as one snapshot of data which will be processed to produce the DOA information. It can be simply expressed as

$$
y_{m}=\sum_{k=1}^{K} A_{k} e^{-j \frac{2 \pi R}{\lambda} \cos \left(\theta_{k}-\frac{2 \pi m}{M}\right)}+\mathcal{E} .
$$

There are many ways to estimate the DOA of $\theta$ in Eq.11. However, because of the appearing of quantity $\frac{2 \pi m}{M}$, we cannot directly use the TFBMP for the DOA estimation similar to [11]. In order to do that, we have to cancel the quantity $\frac{2 \pi m}{M}$ by applying the Beam forming to represent Eq.11. Beam - forming is a term used to describe the process of weighting and summing the output of an array of antennas spatial gain pattern on the array. Phase mode excitation [12], [13] of a circular array is the specific type of Beam forming. For the phase mode excitation based Beam - forming, all weights $\left(w_{m}(s)\right)$, which are in turn assigned to the output of array elements, have the equal magnitude. Meanwhile, the phase of each weight $w_{m}$ is assigned in a linear manner based on the angular position of $m^{\text {th }}$ array element relative to a reference element, array element 0 . The position of a reference element is located in the reception of the plane wave of the UCA depicted in Fig.2.

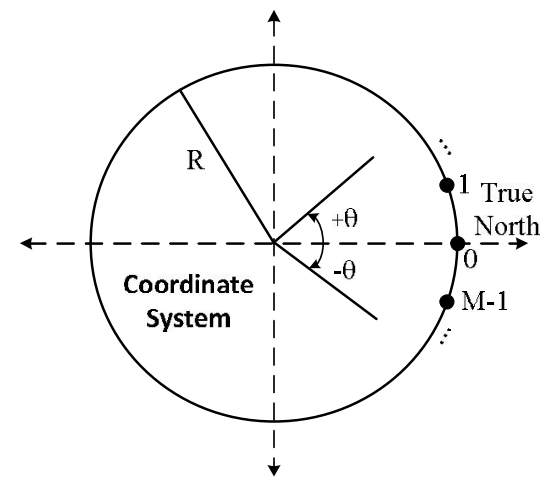

Fig. 2. Reception of plane wave of UCA

In [12], the weight vector that excites the array with phase mode $i$ is mathematically defined as

$$
w_{i}=\frac{1}{M}\left[1 e^{-\frac{j 2 \pi i}{M}} \ldots e^{-\frac{j 2 \pi i m}{M}} \ldots e^{-\frac{j 2 \pi i(M-1)}{M}}\right]^{T} .
$$

By applying the Beam - forming weights to the array - manifold vector of the circular array, the spatial response pattern of the UCA after Beam forming based on excitation of phase mode $i$ of the array can be approximated by

$$
f_{i}(\theta)=w_{i}^{H} a^{c}(\theta) \approx j^{|i|} \mathcal{J}_{|i|}\left(\frac{2 \pi}{\lambda} R\right) e^{j i \theta},
$$

where $a^{c}(\theta)$ is the array - manifold vector with DOA of $\theta$ and $\mathcal{J}_{i}(x)$ is the Bessel function of the first kind of order $i$. Based on [12], we must also note that the number of modes of a given UCA that can be excited is limited. We call the maximum number of modes $N$ and the selection of an appropriate value for the maximum excited mode $N$ satisfies:

$$
N \approx \frac{2 \pi}{\lambda} R .
$$




\section{Research, Development on Information and Communications Technology}

Collecting the weights for all modes with $|i| \leq N$, we define the phase mode excitation beam-forming as

$$
\widetilde{\boldsymbol{F}}_{p i}^{H}=\boldsymbol{C} \boldsymbol{V}^{H}
$$

where

$$
C=\operatorname{diag}\left\{j^{-N}, \ldots, j^{-1}, j^{0}, j^{-1}, \ldots, j^{-N}\right\},
$$

$$
V^{H}=\sqrt{M}\left[w_{-N} \ldots w_{0} \ldots w_{N}\right] .
$$

After applying the Beam - former to the array manifold vector of the UCA, the Beam - space manifold of the UCA can be defined as

$$
\tilde{a}(\theta)=\widetilde{\boldsymbol{F}}_{\boldsymbol{i}}^{H} a^{c}(\theta)=\boldsymbol{C} \boldsymbol{V}^{H} a^{c}(\theta)=\sqrt{M} \boldsymbol{J}_{\xi} c(\theta),
$$

where

$$
\begin{gathered}
\boldsymbol{J}_{\xi}=\operatorname{diag}\left\{\mathcal{J}_{-N}(\xi), \ldots, \mathcal{J}_{0}(\xi), \ldots \mathcal{J}_{N}(\xi)\right\}, \\
\boldsymbol{J}_{\xi}=\operatorname{diag}\left\{\mathcal{J}_{-N}\left(\frac{2 \pi}{\lambda} R\right), \ldots J_{0}\left(\frac{2 \pi}{\lambda} R\right), \ldots \mathcal{J}_{N}\left(\frac{2 \pi}{\lambda} R\right)\right\}, \\
c(\theta)=\left[e^{-j N \theta}, \ldots, e^{-j \theta}, e^{j 0}, e^{j \theta}, \ldots, e^{j N \theta}\right]^{T} .
\end{gathered}
$$

Based on Eq.19, the output signal from antenna array in Beam - space can be rewritten as

$$
\begin{aligned}
\tilde{y}_{m}(n) & =\left[\begin{array}{c}
\tilde{y}_{-N}(n) \\
\vdots \\
\tilde{y}_{N}(n)
\end{array}\right]=\widetilde{\boldsymbol{F}}_{i}^{H} y_{m}(n) \\
& =\sum_{k=1}^{K}\left(\tilde{a}\left(\theta_{k}\right) A_{k}(n)\right)+\widetilde{\mathcal{E}}(n) .
\end{aligned}
$$

According to the Eq.22 and Eq.23, the expression for the observed excitation of single mode $i$ can be expressed as

$$
\tilde{y}_{m, i}(n)=\sum_{k=1}^{K}\left(\mathcal{J}_{|i|}\left(\frac{2 \pi}{\lambda} R\right) e^{j i \theta_{k}} A_{k}(n)\right)+\tilde{\varepsilon}_{m, i}(n),
$$

where $-N \leq i \leq N$.

\section{DOA ESTIMATION USING TOTAL FORWARD - BACKWARD MATRIX PENCIL METHOD}

Assuming that $K$ signals approach the array in presence of white noise, the output signal at each antenna element is presented as in Eq.11. When applying Phase mode excitation Beam - forming, the output signal can be mathematically represented as in Eq.24. Because the Bessel function term $\mathcal{J}_{|i|}\left(\frac{2 \pi}{\lambda} R\right)$ does not depend on $k$ index, we can easily cancel this term by multiplying a normalization coefficient $\Delta i=\left(\mathcal{J}_{|i|}\left(\frac{2 \pi}{\lambda} R\right)\right)^{-1}$ to each element of the Beam space data vector. Therefore, we can get

$$
\bar{y}_{m, i}(n)=\Delta_{m, i} \tilde{y}_{m, i}(n)
$$

$$
=\frac{\tilde{y}_{m, i}(n)}{J_{|i|}\left(\frac{2 \pi}{\lambda} R\right)}=\sum_{k=1}^{K} A_{k}(n) z_{k}^{i}+\bar{\varepsilon}_{m, i}(n),
$$

where $z_{k}=e^{j \theta_{k}}$ and $-N \leq i \leq N$.

We now estimate the DOA information of the output signal as presented in Eq.25.

We need to set $M^{\prime}=2 N+1$ and choose the pencil parameter $L$ with the condition $\frac{M}{3} \leq L \leq \frac{M}{2}$ because of the efficient noise filtering issue described in [5]. Based on TFBMP, we have defined the matrices $\boldsymbol{Y}_{0 f b}$ and $\boldsymbol{Y}_{1 f b}$ as

$$
\begin{aligned}
\boldsymbol{Y}_{0 f b_{2(M \prime-L) \times L}} & =\left[\begin{array}{ccccc}
z_{0} & z_{1} & \cdots & z_{L-2} & z_{L-1} \\
z_{L}^{*} & z_{L-1}^{*} & \cdots & z_{2}^{*} & z_{1}^{*}
\end{array}\right], \\
\boldsymbol{Y}_{1 f b_{2(M \prime-L) \times L}} & =\left[\begin{array}{ccccc}
z_{1} & z_{2} & \cdots & z_{L-1} & z_{L} \\
z_{L-1}^{*} & z_{L-2}^{*} & \cdots & z_{1}^{*} & z_{0}^{*}
\end{array}\right],
\end{aligned}
$$

where ' $*$ ' denotes complex conjugate and $z_{\tau}(\tau=$ $0, \ldots, L)$ is defined as

$$
\begin{aligned}
& \mathrm{z}_{\tau}^{T}=\left[\begin{array}{llll}
y_{\tau} & y_{\tau+1} & \ldots & y_{M^{\prime}-L+\tau-1}
\end{array}\right], \\
& (\tau=0, \ldots, L) \text {. }
\end{aligned}
$$

Based on Eq.26 and Eq.27, the Matrix Pencil, $\boldsymbol{Y}_{1 f b}-z \boldsymbol{Y}_{0 f b}$ ( $z$ is complex scalar), can be built to DOA estimate, but, for noisy data, the best way is to 
perform a Singular Value Decomposition (SVD) on the "all data" matrix. This matrix is given by"

$$
\boldsymbol{Y}_{f b_{2(M \prime-L) \times(L+1)}}\left[\begin{array}{llllll}
z_{0} & z_{1} & \cdots & z_{L-1} & z_{L} \\
z_{L}^{*} & z_{L-1}^{*} & \cdots & z_{1}^{*} & z_{0}^{*}
\end{array}\right] .
$$

It is seen that $\boldsymbol{Y}_{0 f b}$ and $\boldsymbol{Y}_{1 f b}$ are obtained from $\boldsymbol{Y}_{f b}$ by deleting, respectively, its $(L+1)^{t h}$ and first columns

$$
\begin{aligned}
& \boldsymbol{Y}_{f b_{2(M \prime-L) \times(L+1)}}=\left[\boldsymbol{Y}_{\left.0 f b_{2(M \prime-L) \times L}, \mathrm{c}_{\mathrm{L}+1}\right],}\right. \\
& \boldsymbol{Y}_{f b_{2\left(M^{\prime}-L\right) \times(L+1)}}=\left[\mathrm{c}_{1}, \boldsymbol{Y}_{1 f b_{2\left(M^{\prime}-L\right) \times L}}\right] .
\end{aligned}
$$

On the other hand, the SVD of $\boldsymbol{Y}_{f b}$ is

$$
\begin{aligned}
& \boldsymbol{Y}_{f b_{2\left(M^{\prime}-L\right) \times(L+1)}}= \\
& \boldsymbol{U}_{2\left(M^{\prime}-L\right) \times 2\left(M^{\prime}-L\right)} \boldsymbol{\Sigma}_{2\left(M^{\prime}-L\right) \times(L+1)} \boldsymbol{V}_{(L+1) \times(L+1)}^{H},
\end{aligned}
$$

where $H$ denotes complex conjugate transpose of a matrix, $\boldsymbol{U}, \Sigma$, and $\boldsymbol{V}$ are given by

$$
\boldsymbol{\Sigma}=\operatorname{diag}\left\{\sigma_{1}, \sigma_{2} \ldots \sigma_{p}\right\},
$$

where $p=\min \left\{2\left(M^{\prime}-L\right), L+1\right\}$ and $\sigma_{1} \geq \sigma_{2} \geq$ $\cdots \geq \sigma_{p} \geq 0$

$$
\begin{gathered}
\boldsymbol{U}=\left[u_{1}, u_{2}, \ldots, u_{2\left(M^{\prime}-L\right)}\right], \\
\boldsymbol{Y}_{f b}^{H} u_{k}=\sigma_{k} v_{k}, \\
(k=1, \ldots, p) \\
\boldsymbol{V}=\left[v_{1}, v_{2}, \ldots, v_{(L+1)}\right], \\
\boldsymbol{Y}_{f b}^{H} v_{k}=\sigma_{k} u_{k}, \\
(k=1, \ldots, p) \\
\boldsymbol{U}^{H} \boldsymbol{U}=\boldsymbol{I}, \boldsymbol{V}^{H} \boldsymbol{V}=\boldsymbol{I},
\end{gathered}
$$

where $\sigma_{k}$ are the singular values of $\boldsymbol{Y}_{f b}$ and the vector $u_{k}$ and $v_{k}$ are the $k^{\text {th }}$ left and right singular vector, respectively.

The problem can be computationally improved by applying the singular value filtering to obtain the $K$ largest singular values of $\boldsymbol{Y}_{f b}$.

$$
\overline{\boldsymbol{Y}}_{f b_{2\left(M^{\prime}-L\right) \times(L+1)}}=\overline{\boldsymbol{U}}_{2(M \prime-L) \times K} \overline{\boldsymbol{\Sigma}}_{K \times K} \overline{\boldsymbol{V}}_{K \times(L+1)}^{H},
$$

where

$$
\bar{\Sigma}=\operatorname{diag}\left\{\sigma_{1}, \sigma_{2} \ldots \sigma_{K}\right\}
$$

has the $K$ largest singular values of $\boldsymbol{\Sigma}$, and the matrices $\overline{\boldsymbol{U}}$ and $\overline{\boldsymbol{V}}$ are formed by extracting the singular vectors corresponding to $K$ singular values. Extract $\overline{\boldsymbol{V}_{0}}$ and $\overline{\boldsymbol{V}_{\mathbf{1}}}$ from $\overline{\boldsymbol{V}}$ as follows:

$$
\overline{\boldsymbol{V}}=\left[\overline{\boldsymbol{V}}_{\mathbf{0}}, v_{L+1}\right], \overline{\boldsymbol{V}}=\left[v_{1}, \overline{\boldsymbol{V}}_{1}\right] .
$$

Same as above, $\overline{\boldsymbol{Y}}_{0 f b}$ and $\overline{\boldsymbol{Y}}_{1 f b}$ are established as

$$
\begin{gathered}
\overline{\boldsymbol{Y}}_{0 f b}=\overline{\boldsymbol{U}} \overline{\boldsymbol{\Sigma}} \overline{\boldsymbol{V}}_{\mathbf{0}}^{H}, \\
\overline{\boldsymbol{Y}}_{1 f b}=\overline{\boldsymbol{U}} \overline{\boldsymbol{\Sigma}} \overline{\boldsymbol{V}}_{1}^{H} .
\end{gathered}
$$

Now, consider the matrix pencil

$$
\overline{\boldsymbol{Y}}_{1 f b}-z \overline{\boldsymbol{Y}}_{0 f b}
$$

and left multiplying Eq.44 by $\overline{\boldsymbol{Y}}_{0 f b}^{+}$yields

$$
q^{H}\left(\overline{\boldsymbol{Y}}_{1 f b} \overline{\boldsymbol{Y}}_{0 f b}^{+}-z \boldsymbol{I}\right)=0^{H},
$$

where $\overline{\boldsymbol{Y}}_{0 f b}^{+}$is the Moore - Penrose pseudo - inverse of $\boldsymbol{Y}_{0 f b}$,

$$
\overline{\boldsymbol{Y}}_{0 f b}^{+}=\left(\overline{\boldsymbol{V}}_{0}^{H}\right)^{+} \overline{\boldsymbol{\Sigma}}^{-1} \overline{\boldsymbol{U}}^{+} .
$$

Substituting Eq.43 and Eq.46 into Eq.45 the equivalent generalized eigen - problem becomes

$$
q^{H}\left(\overline{\boldsymbol{V}}_{1}^{H}-{ }_{z} \overline{\boldsymbol{V}}_{0}^{H}\right)=0^{H} .
$$

Left multiplying Eq.47 by $\overline{\boldsymbol{V}_{0}}$, we have

$$
q^{H}\left(\overline{\boldsymbol{V}}_{1}^{H} \overline{\boldsymbol{V}}_{0}-z_{\overline{\boldsymbol{V}}_{0}^{H}} \overline{\boldsymbol{V}}_{0}\right)=0^{H} .
$$

Using the values of the generalized eigenvalues, $z$, of Eq.48, angles of arrival can be estimated as

$$
\theta_{k}=\mathfrak{I}\left(\ln \left(z_{k}\right)\right),
$$

Where $\mathfrak{I}\left(\ln \left(z_{k}\right)\right)$ is the imaginary part of $\ln \left(z_{k}\right)$. 


\section{Research, Development on Information and Communications Technology}

\section{SIMULATION RESULTS}

The DF algorithm is simulated using Matlab in order to examine the performance of the algorithm. In this paper, we assume three signals $(K=3)$ impinging on a 10 - element antenna array $(M=10)$ with inter - spacing $d=0.3 \lambda$. The value of $d$ parameter is optimizedin order to reduce the size of the antenna array as well as to ensure the acceptable mutual coupling factor between antenna elements. The simulation is performed in two cases: incoherent and coherent signals.

\section{A. Incoherent signals}

In this paper, three incoherent signals at $1 \mathrm{GHz}$, $1.2 \mathrm{GHz}$ and $1.3 \mathrm{GHz}$ are supposed to impinge on the antenna array at the DOA of $-50^{\circ}, 60^{\circ}$ and $160^{\circ}$, respectively. One of the most significant advantages of TFBMP is that it can extract the DOA information with only one snapshot [14]. The estimated DOAs in the simulation are the numerical values as in Eq.49. However, in order to demonstrate visually the results, we illustrate the DOA in XOY plane, in which the $\mathrm{X}$ - Axis is the DOA of incoming signals and the Y Axis is the indicating factor. This factor is set to 1 corresponding to the estimated DOA in X - Axis. Figure 3 presents the DOA estimation of those signals in the AWGN channel in which the SNR is set to $10 \mathrm{~dB}$. We can see that the DOA of signal of interest is estimated accurately by the proposed algorithm with very small error. In Fig. 4, the simulation is performed in the AWGN channel with the variable SNRs from $-10 \mathrm{~dB}$ to $40 \mathrm{~dB}$. The RMSE is presented to prove the accuracy of this algorithm. It is easy to see that this method is quite good in a white noise environment although with only one snapshot. In similar situation, the DOA information could not be estimated by the Root - Music algorithm as shown in Fig.5. The fact is due to the inaccurate estimate of the correlation matrix.

In order to evaluate the influence of the number of snapshots on the performance of this algorithm, we execute this method with the same inputs as above with 1000 snapshots. The simulation result shown in Fig. 6 indicates that when we increase the number of snapshots, the accuracy of this method increases with RMSE equal to $0.3379^{\circ}$ in comparison with $0.5307^{\circ}$ when one snapshot captured. Moreover, with multi snapshots, this algorithm in the AWGN channel with varying SNRs operates more stably than in case of one snapshot as shown in Fig.7 . Obviously, we can get the better results when we increase the number of snapshots. The simulation result plotted in Fig. 8 has statement proved that. However, it can be observed that when the number of snapshots is more than 90 , the accuracy of the algorithm seems to be invariable. On the other hand, when the number of snapshot is increased, the computation time also significantly increases. Therefore, the trade - off between the computation time and the accuracy of the algorithm should be taken into account.

Furthermore, the angle resolution of this method is also a factor to be assessed. In order to do that we perform this algorithm with some pairs of incoherent incident signals whose SNRs are set to $10 \mathrm{~dB}$. The simulation results are stored in Table 1. If we consider the RMSE of desired results less than 2 degrees, from Table 1, we can easily see that for the case of incoherent incoming signals, this method will work well with the resolution approximately 5 degrees.

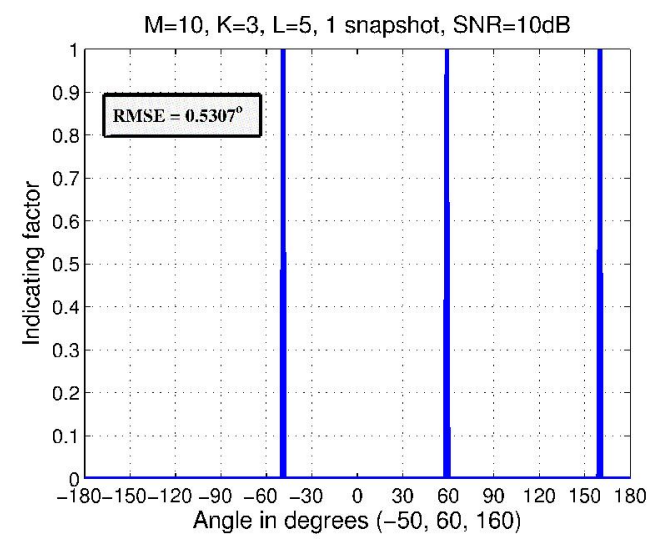

Fig.3. DOA estimation results of three incoherent signals at the DOA of $-50^{\circ}, 60^{\circ}$ and $160^{\circ}$ with one snapshot. 


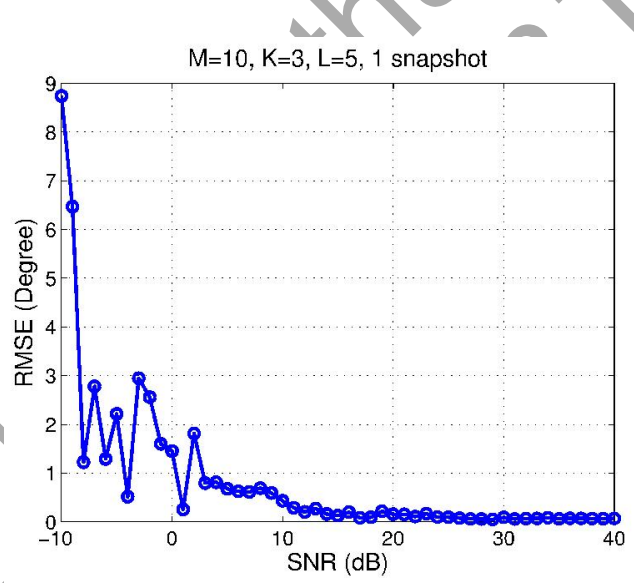

Fig.4. Estimation accuracy at the DOA of $-50^{\circ}, 60^{\circ}$ and $160^{\circ}$ of three incoherent signals with one snapshot

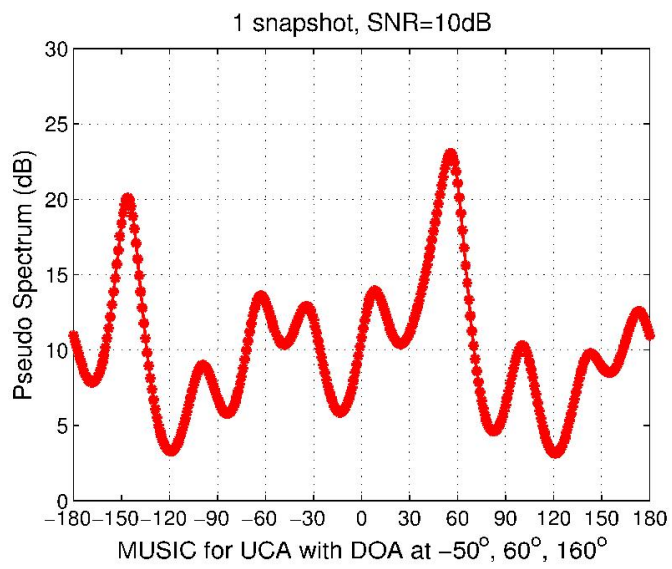

Fig.5. DOA estimation using Root - MUSIC with one snapshot

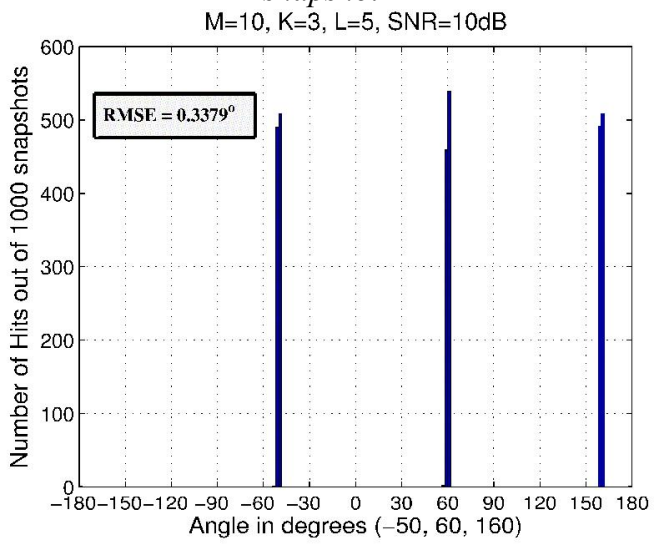

Fig.6. 1000 snapshots simulation results of three incoherent signals at the DOA of $-50^{\circ}, 60^{\circ}$ and $160^{\circ}$

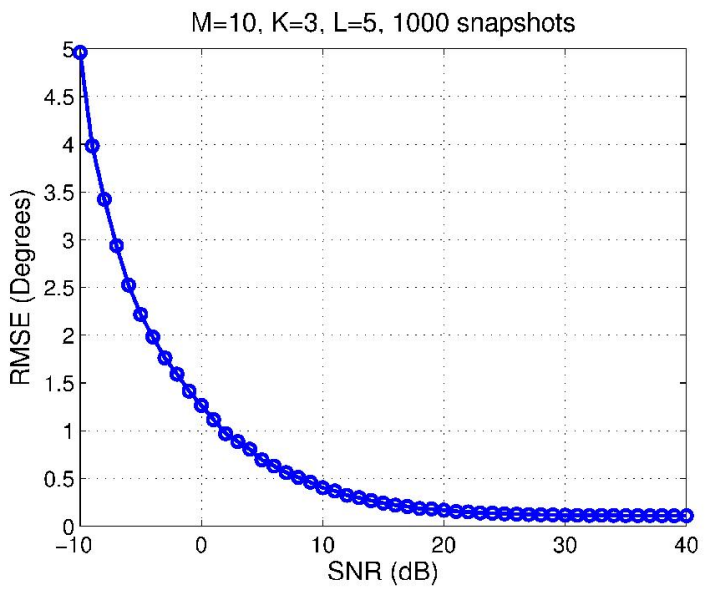

Fig. 7. Estimation accuracy at the DOA of $-50^{\circ}, 60^{\circ}$ and $160^{\circ}$ of three incoherent signals with 1000 snapshots

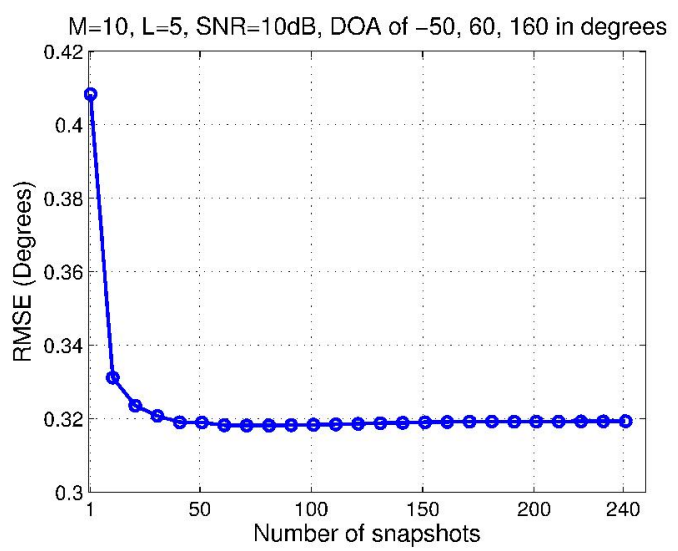

Fig. 8. DOA estimation with the different number of snapshots

Table 1. Angle resolution for incoherent signals

\begin{tabular}{|c|c|c|c|c|}
\hline$\theta_{1}$ & $\theta_{2}$ & \multicolumn{2}{|c|}{ DOA estimation results } & RMSE \\
\hline $30^{\circ}$ & $31^{\circ}$ & $30.5955^{\circ}$ & $96.9867^{\circ}$ & $46.6615^{\circ}$ \\
\hline $30^{\circ}$ & $32^{\circ}$ & $31.0707^{\circ}$ & $43.0304^{\circ}$ & $7.8363^{\circ}$ \\
\hline $30^{\circ}$ & $33^{\circ}$ & $31.7978^{\circ}$ & $42.0791^{\circ}$ & $6.5446^{\circ}$ \\
\hline $30^{\circ}$ & $34^{\circ}$ & $32.1605^{\circ}$ & $36.9469^{\circ}$ & $2.5838^{\circ}$ \\
\hline $30^{\circ}$ & $35^{\circ}$ & $31.1450^{\circ}$ & $34.2202^{\circ}$ & $0.9796^{\circ}$ \\
\hline $30^{\circ}$ & $36^{\circ}$ & $30.7914^{\circ}$ & $35.2331^{\circ}$ & $0.7793^{\circ}$ \\
\hline $30^{\circ}$ & $37^{\circ}$ & $30.3921^{\circ}$ & $36.2355^{\circ}$ & $0.6075^{\circ}$ \\
\hline $30^{\circ}$ & $38^{\circ}$ & $30.3557^{\circ}$ & $37.1282^{\circ}$ & $0.6658^{\circ}$ \\
\hline
\end{tabular}

B. Coherent signals

Another remarkable advantage of TFBMP is that it can extract the DOA information of coherent incoming signals in highly correlative environment 


\section{Research, Development on Information and Communications Technology}

[15], which could not be found by the Root - MUSIC algorithm as shown in Fig.9. In this case, the algorithm has to break the correlation between the signals and then estimates and produces the DOA information. In order to assess performance of the algorithm, we execute this algorithm in similar situations with the case of incoherent signals. Figure 10 demonstrates the simulation results of three coherent signals at $1 \mathrm{GHz}$, which come from the DOA of $-30^{\circ}, 0^{\circ}$ and $110^{\circ}$ with one snapshot.

Figure 11 shows the accuracy of DOA estimation according to the variable SNRs. And the relation between the accuracy of the algorithm and number of snapshots is shown in Fig.12. We can see that the DOA information of the coherent incoming signals can still be exactly calculated by this algorithm. However, the performance of the algorithm is less than the incoherent case. In this case, the RMSE increases and we need at least 150 snapshots, the accuracy could be invariable. This phenomenon is due to the characteristic of correlation signals.

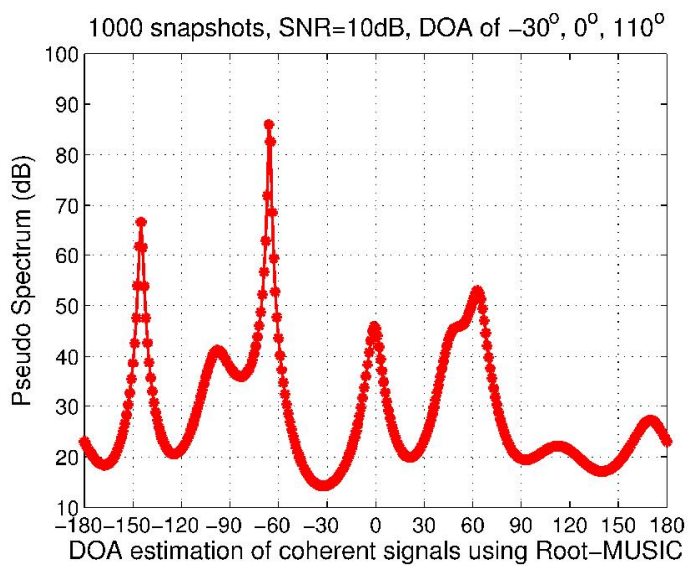

Fig.9. DOA estimation of three coherent signals in highly correlative environment at $1 \mathrm{GHz}$ from $-30^{\circ}, 0^{\circ}$ and $110^{\circ}$ using Root-MUSIC

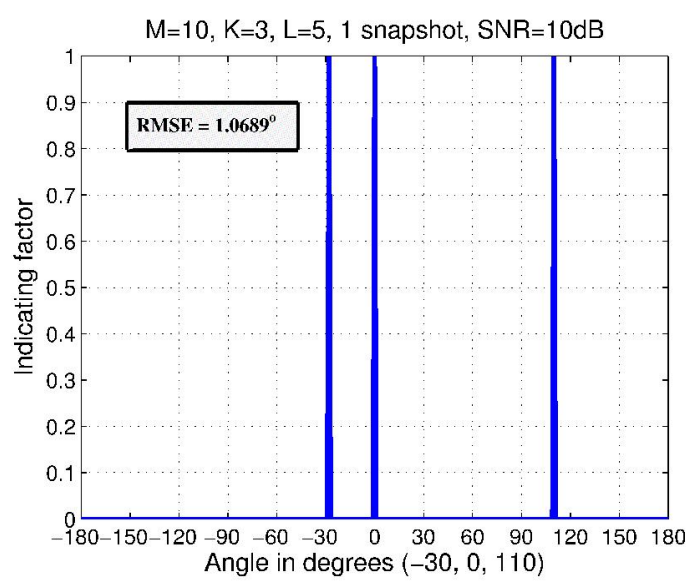

Fig.10. DOA estimation of three coherent signals at $1 \mathrm{GHz}$

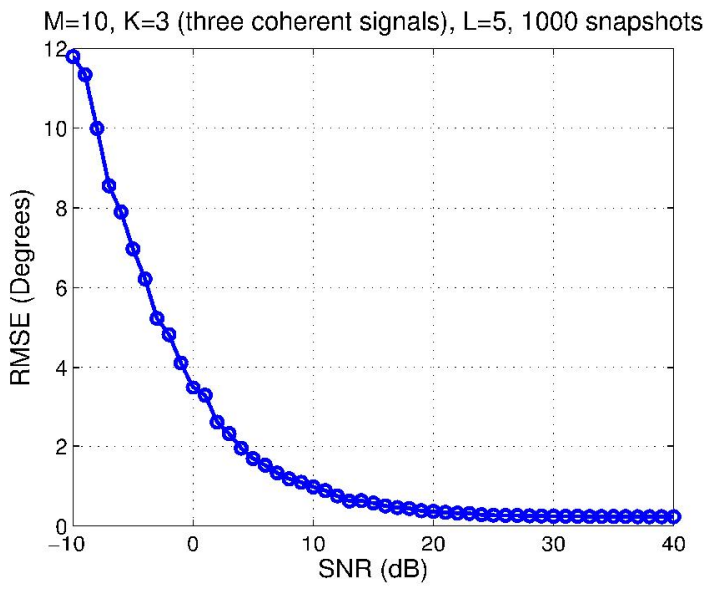

Fig. 11. Estimation accuracy at the DOA of $-30^{\circ}$,

$0^{\circ}$ and $110^{\circ}$ of three coherent signals with 1000 snapshots

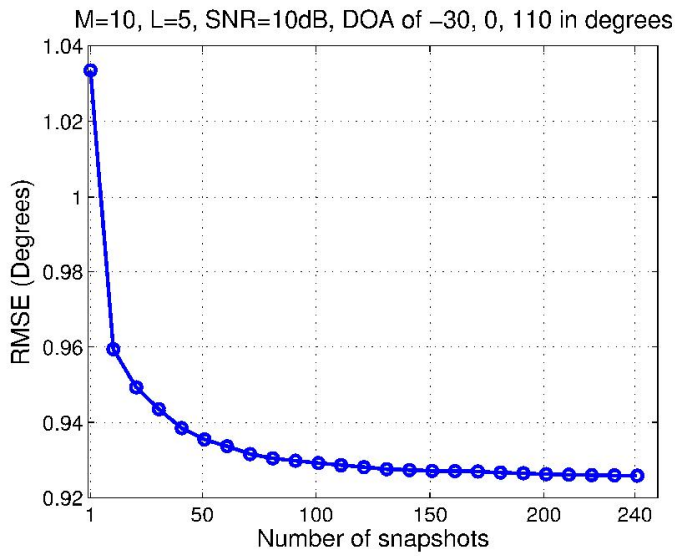

Fig. 12. DOA estimation with the different number of snapshots (in case of coherent signals) 
Moreover, the angle resolution is the factor to compare the performace of the algorithm between two cases. In order to do that we perform this algorithm with the same value inputs of DOA as in the incoherent case. The simulation results are stored in Table 2. If we still consider the RMSE of desired results less than 2 degrees, from Table 2, we can easily see that this method will work well with the resolution of approximately 8 degrees. Evidently, the angle resolution of this method in this case decreases.

Table 2. Angle resolution for coherent signals

\begin{tabular}{|c|c|c|c|c|}
\hline$\theta_{1}$ & $\theta_{2}$ & \multicolumn{4}{|c|}{ DOA estimation results } & RMSE \\
\hline $30^{\circ}$ & $31^{\circ}$ & $30.5184^{\circ}$ & $-7.0974^{\circ}$ & $26.2341^{\circ}$ \\
\hline $30^{\circ}$ & $32^{\circ}$ & $30.7883^{\circ}$ & $5.8804^{\circ}$ & $17.0766^{\circ}$ \\
\hline $30^{\circ}$ & $33^{\circ}$ & $31.7590^{\circ}$ & $-31.4549^{\circ}$ & $43.4640^{\circ}$ \\
\hline $30^{\circ}$ & $34^{\circ}$ & $31.4443^{\circ}$ & $61.6602^{\circ}$ & $19.5854^{\circ}$ \\
\hline $30^{\circ}$ & $35^{\circ}$ & $32.0660^{\circ}$ & $39.7760^{\circ}$ & $3.6795^{\circ}$ \\
\hline $30^{\circ}$ & $36^{\circ}$ & $31.2046^{\circ}$ & $39.7031^{\circ}$ & $2.7535^{\circ}$ \\
\hline $30^{\circ}$ & $37^{\circ}$ & $31.8203^{\circ}$ & $40.0928^{\circ}$ & $2.5376^{\circ}$ \\
\hline $30^{\circ}$ & $38^{\circ}$ & $30.8867^{\circ}$ & $39.4569^{\circ}$ & $1.2060^{\circ}$ \\
\hline $30^{\circ}$ & $39^{\circ}$ & $28.6920^{\circ}$ & $38.2658^{\circ}$ & $1.0606^{\circ}$ \\
\hline $30^{\circ}$ & $40^{\circ}$ & $28.8908^{\circ}$ & $39.6352^{\circ}$ & $0.9038^{\circ}$ \\
\hline
\end{tabular}

\section{CONCLUSIONS}

We propose, in this paper, an efficient approach for DOA estimation by using Total Forward Backward Matrix Pencil algorithm. The benefit of this approach is that it can estimate correctly the DOA of coherent incoming signals because it deals directly with the input samples of signal and does not require the estimation of a correlation matrix to produce DOA information. Moreover, this algorithm can operate with only one discrete time sample of signal, which means that the sampling frequency can be considerably reduced. In this way, the computational complexity significantly decreases in comparison with the other algorithms. Obviously, this method is a high - performance DOA estimation algorithm. It is one of the practical ways to estimate the DOA information of the radio frequency signals in the presence of noise.

\section{ACKNOWLEDGEMENT}

This research is carried out in the framework of the project titled "Design and Implementation of an Earth Station based on Software Defined Radio in the satellite communication system" in the national program on space technology under the grant number $\mathrm{VT} / \mathrm{CN}-02 / 14-15$. This research is funded by the Vietnam Academy of Science and Technology. The authors would like to thank the Vietnam Academy of Science and Technology and the Ministry of Science and Technology, Vietnam for their financial support.

\section{REFERENCES}

[1] RDF Products, "Basics of the watson-watt radio direction finding technique,"in Web Note $\mathrm{WN}-002$, December 1998.

[2] D. Peavey and T. Ogunfunmi, "The single channel interferometer using a pseudo-doppler direction finding system," in Proc. IEEE Conference on Acoustics, Speech, and Signal Processing, April 1997, vol. 5, pp. 4129-4132.

[3] H. T. Thanh, T. N. Ha, and V. V. Yem, "Novel direction finding algorithm based on phase locked loop with low computational complexity," in Proc. International Conference on Advanced Technologies for Communications (ATC), Ho Chi Minh, Vietnam, October 2013, pp. 437 - 442.

[4] J.-H. C. C.-S. Park and S.-P. N.-W. Jang, "A MultiChannel Correlative Vector Direction Finding System Using Active Dipole Antenna Array for Mobile Direction Finding Applications,"Journal of The Korea Electromagnetic Engineering Society, vol. 7, 2007.

[5] Y. Hua and T. Sarkar, "Matrix pencil method for estimating parameters of exponentially damped/undamped sinusoids in noise," IEEE Transactions on Acoustics, Speech, and Signal Processing, vol. 38, No. 5, pp. 814-824, May 1990.

[6] R. S. Adve, T. K. Sarkar, O. M. Pereira-Filho, and S. M. Rao, "Extrapolation of time-domain responses from three-dimensional conducting objects utilizing the matrix pencil technique,"IEEE Transactions on Antennas and Propagation, vol. 45, pp. 147-156, 1997.

[7] C. Lau, R.S.Adve, and T. Sarkar, "Minimum norm mutual coupling compensation with applications in direction of arrival estimation," IEEE Transactions on Antennas and Propagation, vol. 52, Issue 8, pp. 2034 2041, August 2004.

[8] N. Dharamdial, R. Adve, and R. Farha, "Multipath delay estimations using matrix pencil," in Proc. 


\section{Research, Development on Information and Communications Technology}

Wireless Communications and Networking Conference (WCNC), March 2003, vol.1, pp. 632-635.

[9] R. O. Schmidt, "Multiple emitter location and signal parameter estimation," IEEE Transactions on Antennas and Propagation, vol. 34, pp. 276-280, 1986.

[10] J. E. F. del Rio and T. K. Sarkar, "Comparison between the matrix pencil method and the fourier transform technique for high-resolution spectral estimation," in Digital Signal Processing, vol. 6, No.0011, pp. 108-125, 1996.

[11]H. T. Thanh, V. V. Yem, N. D. Minh, and H. D. Thang, "Direction of Arrival estimation using the Total Forward - Backward Matrix Pencil Method," in Proc.International Conference on Communications and Electronics (ICCE), Danang, Vietnam, 2014, pp.

\section{$718-722$.}

[12]C. P. Mathews and M. D. Zoltowski, "Eigenstructure techniques for 2-D angle estimation with uniform circular arrays,"IEEE Transactions on Signal Processing, vol. 42, pp. 2395-2407, 1994.

[13] J. G. Davis and A. A. P. Gibson, "Phase Mode Excitation in Beamforming Arrays," in 3rd European Radar Conference(EuRAD), September 2006, pp. 307310,

[14] N. Yilmazer, T. K. Sarkar, and M. Salazar-Palma, "DOA Estimation using Matrix Pencil and ESPRIT methods using single and multiple snapshots," in 2010 URSI International Symposium on Electromagnetic Theory (EMTS), 2010, pp. 215-218.

[15] U.Somalatha, T.V.S.Gowtham Prasad and T. Ravi Kumar Naidu, "DOA Estimation of Uncorrelated and Coherent Signals in Multipath Environment Using ULA Antennas", International Journal of Innovative Research in Computer and Communication Engineering, Vol.2, Special Issue 4, pp 47 - 55, September 2014.

\section{AUTHORS' BIOGRAPHIES}

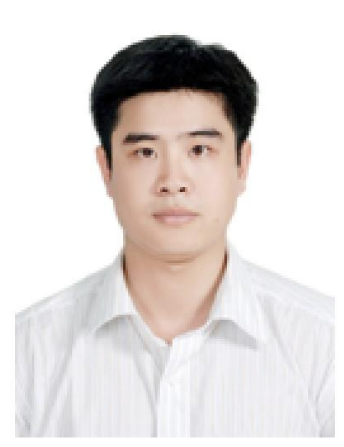

Han Trong Thanh received the Diplomat of Engineer in 2008 and Master of Science in 2010 from the School of Electronics and Telecommunications, Hanoi University of Science and Technology, Vietnam. Currently, he is a Ph.D candidate at Dept. of Communication Engineering, School of Electronics and Telecommunications, HUST. His research interests are localization, signal processing for antenna array and Design of Satellite Navigation Receiver based on Software Defined Radio.

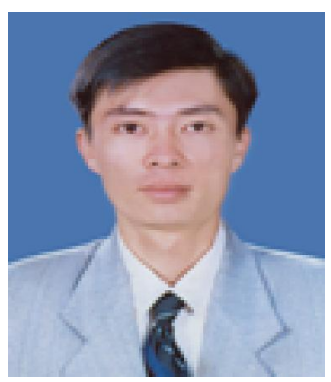

Do Trong Tuan received B.E., M.E., and Dr. Eng. degrees in Electronics and Telecommuni-cations from Hanoi University of Science and Technology in 1997, 2000 and 2007, respectively. $\mathrm{He}$ is now senior lecturer at Department of Aerospace Electronics, School of Electronics and Telecom-munications, Hanoi University of Science and Technology. His interested research themes are Real-time Communication Quality Assurance, Electronic Localization and Navigations, Visible Light and Free Space Optical Communications, Smart Integrated Systems and Cellular Neural Networks.

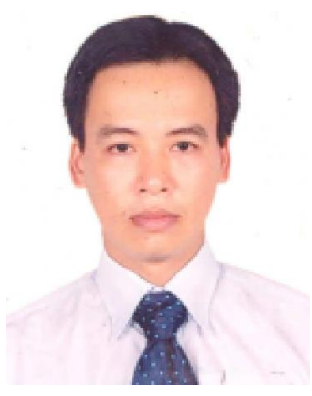

Nguyen Trong Duc received the Ph.D. degree in communications from Laboratory LAHC, Institute Microelectronic Electromagnetic and Photonic, University of Grenoble, France in 2012. Currently, he is the vice dean of the Faculty of Information Technology,

Vietnam Maritime University. His areas of expertise are multi-antenna communication and localization systems, microstrip antennas and antenna array signal processing, embedded systems and intelligence control system.

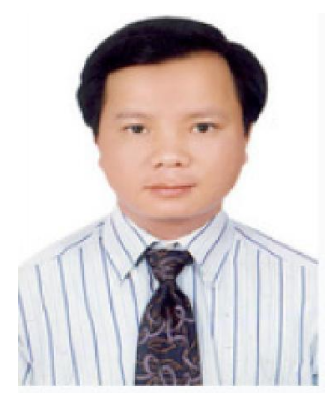

Vu Van Yem received Ph.D. degree from the Department of Electronics and Communications, TELLECOM ParisTech (Formerly ENST Paris), France, in 2005. From 2006 to 2007, he was a postdoctoral researcher at the Department of Hyper-Frequency, the Institute of Electronics and Microelectronics and Nano- 
technology (IEMN), France. He has been qualified as associate professor since 2009 . He is the Head of the Department of Telecommunication Systems, School of Electronics and Telecommunications as well as the Vice-Dean of Graduate School, Hanoi University of Science and Technology, Vietnam.

Dr. Vu Van Yem is a member of national committee on space technology, a member of the Association of Vietnamese Scientists and Experts (AVSE), IEEE and REV.

His research interests are microwave engineering, antenna, chaos-based digital communications as well as adyanced wireless communication and localization systems. 